\title{
Towards Efficient and Compact Phenomenological Representation of Arbitrary Bidirectional Surface Reflectance
}

\author{
Shireen Y. Elhabian \\ syelha01@cardmail.louisville.edu \\ Ham Rara \\ hmrara01@cardmail.louisville.edu
}

\author{
CVIP Lab \\ University of Louisville \\ Louisville, USA \\ http://www.cvip.uofl.edu
}

\author{
Aly A. Farag \\ aly.farag@louisville.edu
}

\begin{abstract}
The visual appearance of real-world surfaces is the net result of surface reflectance characteristics when exposed to illumination. Appearance models can be constructed using phenomenological models which capture surface appearance through mathematical modeling of the reflection process. This yields an integral equation, known as reflectance equation, describing the surface radiance, which depends on the interaction between the incident light field and the surface bidirectional reflectance distribution function (BRDF). The BRDF is a function defined on the cartesian product of two hemispheres corresponding to the incident and outgoing directions; the natural way to represent such a hemispherical function is to use hemispherical basis. However, due to their compactness in the frequency space, spherical harmonics $(\mathrm{SH})$ have been extensively used for this purpose. In this paper, we address the geometrical compliance of hemispherical basis for representing surface BRDF. We propose a tensor product of the hemispherical harmonics $(\mathrm{HSH})$ to provide a compact and efficient representation for arbitrary BRDFs, while satisfying the Helmholtz reciprocity property. We provide an analytical analysis and experimental justification that for a given approximation order, our proposed hemispherical basis provide better approximation accuracy when compared to Zernike-based basis, while avoiding the high computational complexity inherited from such polynomials. We validate our proposed Helmholtz HSH-based basis functions on Oren-Nayar and Cook-Torrance BRDF physical models.
\end{abstract}

\section{Introduction}

Various types of surfaces can be identifiable by their appearance, which is the net result of the surface reflectance characteristics when exposed to illumination. There are three fundamental ways to construct appearance models; (1) understanding the underlying physical process of light-surface interaction; leading to Fresnel's equations, (2) understanding the structure of the surface's material at a micro-scale level, where layered models can be investigated, and (3) using phenomenological models to capture surface appearance through mathematical modeling of the reflection process, leading to the reflectance equation. The theoretical formulation and modeling of such an equation is of a great interest to numerous computer vision tasks, such as illumination modeling [ $\square$ ], surface reflectivity estimation/analysis [ $\square$ ] 


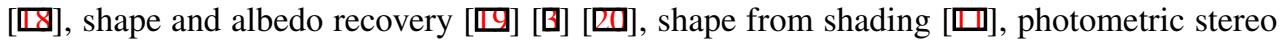
[四], object detection and recognition [미, to name a few.



Figure 1: (a) An object's surface is illuminated by a distant point light source. We use the mean shape from USF HumanID 3D face database [ $\mathbb{}$ ] for illustration. (b) An in-depth view of a surface patch showing surface normals at each surface point. (c) A zoom-in view at a surface point $\mathbf{x}$ seeing its surrounding world through a unit hemisphere $\Omega$ centered at the point and oriented by the surface normal $\mathbf{n}(\mathbf{x})$ at that point. (d) Visualization of up-to 3rd order of our proposed Helmholtz surface reflectance basis at an incident direction $\mathbf{u}_{i}=(\pi / 3,0)$.

Using geometric optics approximation, the reflectance equation, under the assumption of no surface emittance, is an integral equation describing the surface reflected radiance, resulting from the interaction between the incident light field and the surface bidirectional reflectance distribution function (BRDF), which is a mathematical and physical abstraction that captures the positional and directional dependencies of reflection, and in the meantime, hides the complexity of the interaction of light with matter. It is defined as the ratio of the surface radiance in an outgoing direction to the irradiance coming from an incident direction at the same surface point, see Fig. 1. According to its geometrical nature, the BRDF is known to be invariant to permutation of incident and outgoing light directions, which is a property known as Helmholtz reciprocity.

A BRDF can be obtained by (1) deriving an analytic formula based on physical principles, (2) simulating an assumed surface micro-geometry model, or (3) measuring BRDF values based on empirical observation. In all cases, it is required to devise a method for BRDF representation which maintains the Helmholtz reciprocity property. A tabular representation can be used to represent an arbitrary BRDF, however due to its four-dimensionality, full BRDF data are very scarce where the measurements can be restricted to the plane of incidence. At the other end of the spectrum, analytic formula can present a very compact model for BRDF representation, e.g. Cook-Torrance [ $[$ ] and Oren-Nayar [ $\square]$ ]. Despite of the representation compactness, the lack of generality is the main drawback for such models, where there is no guarantee that these analytical models can represent arbitrary measured reflectance data.

On the middle ground, an arbitrary BRDF can be represented as a linear combination of a complete set of orthonormal basis functions, analogous to Fourier basis representing functions over the real line. The BRDF is a function defined on the cartesian product of two hemispheres corresponding to the incident and outgoing directions; the natural way to represent such a hemispherical function is to use hemispherical basis. However, due to their 
compactness in the frequency space, spherical harmonics (SH) have been extensively used


over the cartesian product of two spheres, taking the advantage of symmetry and reciprocity to reduce the non-zero coefficients representing the BRDF. Ramamoorthi [ष]], on the other hand, used two successive SH transformations corresponding to the incoming and outgoing spheres, respectively. Along the same line, Gautron et al. [Q] used two hemispherical harmonics (HSH) transformations to define hemispherical basis. Nonetheless, such definitions

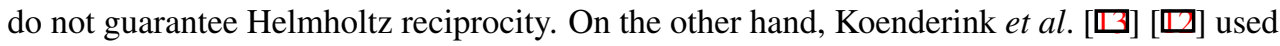
Zernike polynomials [ $\square]$ to define hemispherical basis that satisfy such a property, however such polynomials are known to have high computational complexity [ $\mathrm{\theta}]$ and in the meantime, they are not defined for all combinations of polynomial orders and degrees.

In this paper, we address the geometrical compliance of hemispherical basis for the representation of surface BRDF. In contrast to Gautron et al. [ $Q$ ], we propose a tensor product of the hemispherical harmonics to provide a compact and efficient representation for arbitrary BRDFs, while satisfying the Helmholtz reciprocity property. Since the set of associated Legendre polynomials is distinguished by the property that it contains a polynomial for every combination of order and degree [ $⿴ 囗 ⿱ 一 一]]$, compared to Zernike polynomials which are restricted to even differences between polynomial order and degree, we provide an analytical analysis and experimental justification that for a given approximation order, our proposed Helmhholtz HSH-based basis provide better approximation accuracy when compared to the basis proposed by Koenderink et al. [ㅁ] [ [0], while avoiding the high computational complexity inherited from Zernike polynomials. We believe that our BRDF representation can be used in place of simple Lambertian models in algorithms such as shape-from-shading and photometric stereo. We validate our basis functions on Oren-Nayar and Cook-Torrance BRDF physical models. Scattered BRDF measurements which might violate the Helmholtz reciprocity property can be filtered out through the process of projecting them on the subspace spanned by our HSH-based basis, where the reciprocity property is preserved in the leastsquares sense.

The rest of the paper is organized as follows: Section 2 discusses briefly the surface reflectance function. Sections 3 describes the hemispherical basis of a single hemisphere. Section 4 introduces our proposed orthonormal BRDF basis. Section 5 investigates the representation power of these basis to provide compact description of BRDF physical models. Section 6 concludes this paper.

\section{Surface Reflectance Function}

Under the assumption of no surface emittance, a surface point $\mathbf{x}$ is locally seeing the surrounding world through a unit hemisphere $\Omega$ oriented by the surface normal $\mathbf{n}(\mathbf{x})$ at this point, see Fig. 1. When it is illuminated by radiance $L\left(\mathbf{x}, \mathbf{u}_{i}\right)$ coming in from a directional region of solid angle $d \omega_{i}$ at direction $\mathbf{u}_{i}=\left(\theta_{i}, \phi_{i}\right)$, it receives irradiance $L\left(\mathbf{x}, \mathbf{u}_{i}\right) \cos \theta_{i} d \omega_{i}$, where $\cos \theta_{i}$ accounts for the foreshortening effect, $\theta_{i}, \theta_{o} \in[0, \pi / 2]$ and $\phi_{i}, \phi_{o} \in[0,2 \pi]$. The BRDF, denoted by $\rho_{b d}\left(\mathbf{x}, \mathbf{u}_{i}, \mathbf{u}_{o}\right)$ is the ratio of the surface radiance in the outgoing direction $\mathbf{u}_{o}$ to the irradiance coming from the incident direction $\mathbf{u}_{i}$ at the same surface point. Assuming homogeneous surfaces having position/shift-invariant BRDFs, the spatial parameter can be excluded from the BRDF definition, which becomes mapping the cartesian product of two hemispheres to the real line, i.e. $\rho_{b d}\left(\mathbf{u}_{i}, \mathbf{u}_{o}\right): \Omega \times \Omega \rightarrow \mathbb{R}$. Thus the surface reflectance function, defined as the radiance leaving a surface at a point $\mathbf{x}$ due to irradiance received over the whole incoming upper hemisphere, can be obtained by integrating irradiance-weighted BRDF over all incoming directions. 


$$
\mathscr{R}\left(\mathbf{x}, \mathbf{u}_{o}\right)=\int_{\Omega_{i}} L\left(\mathbf{u}_{i}\right) \rho_{b d}\left(\mathbf{u}_{i}, \mathbf{u}_{o}\right) \cos \theta_{i} d \omega_{i}
$$

This equation lends itself to the image irradiance equation formulated by Horn in [س]].

\section{Hemispherical Complete, Orthonormal Basis}

In order to construct a hemispherical basis on the cartesian product of two hemispheres, the key ingredient is defining a complete set of orthonormal basis for a single hemisphere. Several hemispherical basis have been proposed in literature to represent hemispherical functions. Sloan et al. [R] used SH to represent an even-reflected (about $x y$-plane) version of a hemispherical function. Coefficients were found using least squares SH, however this leads to non-zero values in the lower hemisphere. Koenderink et al. [미 [ㅁ] used Zernike polynomials [ $\square]$, which are basis functions defined on a disk, to build hemispherical basis. Yet, such polynomials have high computational cost, when compared to other polynomials such as the associated Legendre polynomials [ $\mathrm{Q}]$. Makhotkin [四] and Gautron et al. [日] proposed hemispherically orthonomal basis through mapping the negative pole of the sphere to the border of the hemisphere. Such contraction was achieved through shifting the adjoint

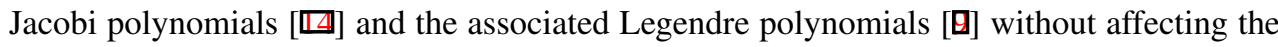
orthogonality relationship. Gautron et al. [Q] sampled the outgoing hemisphere and used hemispherical harmonics to represent the incoming hemisphere corresponding to each outgoing direction. The limitation of such a definition is that it does not satisfy Helmholtz reciprocity.

Makhotkin [ㅁ] outlined a generic approach for defining harmonic basis functions, where the Legendre polynomials $P_{n}(z)$ are chosen, then differentiated $m$-times to define associated Legendre polynomials $P_{n}^{m}(z)$ which are defined by the differential equation (2), and eventually the harmonics basis functions are constructed by combining $P_{n}^{m}(z)$ with $\{\cos (m \phi), \sin (m \phi)\}$.

$$
P_{n}^{m}(z)=\frac{\left(1-z^{2}\right)^{m / 2}}{2^{n} n !} \frac{d^{m+n}}{d z^{m+n}}\left(z^{2}-1\right)^{n}
$$

with order $n \geq 0$ and degree $m \in[0, n]$. Gautron et al $[\theta]$ defined hemispherical basis functions as an adapted version of spherical basis by shifting the associated Legendre polynomials, which remains orthogonal but with different normalization factor. The main idea behind this shifting is limiting the domain of the elevation angle $\theta$ to $\left[0, \frac{\pi}{2}\right]$ when substituting $z$ by $\cos \theta$. Since we are dealing real-valued signal, we use the real-form of $\mathrm{HSH}$, where, analogical to SH definition, the real HSH driven from their complex counterparts are given by [ $\mathbb{}$ ] [ [Q],

$$
H_{n}^{m}(\theta, \phi)=\widetilde{\Theta}_{n}^{m}(\theta) \Phi_{m}(\phi)
$$

with $\theta \in[0, \pi / 2], \phi \in[0,2 \pi]$ and the polar part is defined as $\widetilde{\Theta}_{n}^{m}(\theta)=\widetilde{N}_{n}^{|m|} \widetilde{P}_{n}^{|m|}(\cos \theta)$, where $\widetilde{P}_{n}^{m}(z)=P_{n}^{m}(2 z-1)$, with the normalization factor for $m \geq 0$,

$$
\widetilde{N}_{n}^{m}=\sqrt{\frac{(2 n+1)(n-m) !}{2 \pi(n+m) !}}
$$

where the order $n \geq 0$, the degree $m \in[-n, n]$. and the azimuthal part is defined as,

$$
\Phi_{m}(\phi)= \begin{cases}\sqrt{2} \cos (m \phi) & m>0 \\ 1 & m=0 \\ (-1)^{m} \sqrt{2} \sin (-m \phi) & m<0\end{cases}
$$

By construction, this hemispherical basis are orthonormal satisfying,

$$
\int_{\Omega} H_{n}^{m}(\theta, \phi) H_{n^{\prime}}^{m^{\prime}}(\theta, \phi) d \omega=\delta_{n n^{\prime}} \delta_{m m^{\prime}}
$$

The main advantage of this hemispherical basis definition over the Zernike basis, besides low computational complexity, is that Zernike polynomials are restricted to orders and degrees satisfying $(n-|m|) \bmod 2=0$. 


\section{Helmholtz HSH-based Bidirectional Reflectance Basis}

Consider the product of two HSH basis functions to give a mapping $\Omega \times \Omega \rightarrow \mathbb{R}$ from the tensor product of two hemispheres to the real line, it is possible to define a combined basis function $H_{n}^{m}\left(\mathbf{u}_{i}\right) H_{p}^{q}\left(\mathbf{u}_{o}\right)$. However, in order to preserve the Helmholtz reciprocity property, we define what we call Helmholtz HSH-based basis by symmetrizing the combined basis with respect to the incident and outgoing directions.

$$
\mathscr{H}_{n p}^{m q}\left(\mathbf{u}_{i}, \mathbf{u}_{o}\right)=N_{n p}^{m q}\left(H_{n}^{m}\left(\mathbf{u}_{i}\right) H_{p}^{q}\left(\mathbf{u}_{o}\right)+H_{p}^{q}\left(\mathbf{u}_{i}\right) H_{n}^{m}\left(\mathbf{u}_{o}\right)\right)
$$

where $N_{n p}^{m q}$ is a normalization factor which guarantee the basis orthonormality, using the orthonormality of HSH basis (6), it can be defined as,

$$
N_{n p}^{m q}=\frac{1}{\sqrt{2+2 \delta_{n p} \delta_{m q}}}
$$

Thus, an arbitrary BRDF can be represented in terms of the Helmholtz HSH-based basis as follows,

$$
\rho_{b d}\left(\mathbf{u}_{i}, \mathbf{u}_{o}\right)=\sum_{n=0}^{\infty} \sum_{p=0}^{\infty} \sum_{m=-n}^{n} \sum_{q=-p}^{p} a_{n p}^{m q} \mathscr{H}_{n p}^{m q}\left(\mathbf{u}_{i}, \mathbf{u}_{o}\right)
$$

where the expansion coefficients can be defined as ${ }^{1}$,

$$
a_{n p}^{m q}=\int_{\Omega_{o}} \int_{\Omega_{i}} \rho_{b d}\left(\mathbf{u}_{i}, \mathbf{u}_{o}\right) \mathscr{H}_{n p}^{m q}\left(\mathbf{u}_{i}, \mathbf{u}_{o}\right) d \omega_{i} d \omega_{o}
$$

In order to translate the four-dimensional problem into a two-dimensional one, for a fixed incident direction, the BRDF is a hemispherical function of the outgoing direction, thus to obtain a better insight on the representation power of the proposed basis and how the approximation order affects the function reconstruction accuracy, it is helpful to derive the basis in such a frame. For a fixed incident direction $\mathbf{u}_{i}^{o}$, we have; if $n=0$ and $m=0^{2}$,

$$
a_{0 p}^{0 q}= \begin{cases}\sqrt{2 \pi} N_{0 p}^{0 q} \int_{\Omega_{o}} \rho_{b d}\left(\mathbf{u}_{i}^{o}, \mathbf{u}_{o}\right) H_{p}^{q}\left(\mathbf{u}_{o}\right) d \omega_{o} & p \neq 0, q \neq 0 \\ 2 N_{00}^{00} \int_{\Omega_{o}} \rho_{b d}\left(\mathbf{u}_{i}^{o}, \mathbf{u}_{o}\right) d \omega_{o} & p=0, q=0\end{cases}
$$

and if $n \neq 0$ and $m \neq 0$,

$$
a_{n p}^{m q}= \begin{cases}\sqrt{2 \pi} N_{n 0}^{m 0} \int_{\Omega_{o}} \rho_{b d}\left(\mathbf{u}_{i}^{o}, \mathbf{u}_{o}\right) H_{n}^{m}\left(\mathbf{u}_{o}\right) d \omega_{o} & p=0, q=0 \\ 0 & p \neq 0, q \neq 0\end{cases}
$$

We can observe that the reciprocity property is also maintained in the expansion coefficients, where $a_{p 0}^{q 0}=a_{0 p}^{0 q}$. Using this obervation, the BRDF can be represented in terms of the expansion coefficients as follows, (proof is omitted due to lack of space).

$$
\rho_{b d}\left(\mathbf{u}_{i}^{o}, \mathbf{u}_{o}\right)=\frac{1}{\pi} a_{00}^{00} N_{00}^{00}+\sqrt{\frac{2}{\pi}} \sum_{p=1}^{\infty} \sum_{q=-p}^{p} a_{0 p}^{0 q} N_{0 p}^{0 q}\left(H_{p}^{q}\left(\mathbf{u}_{i}^{o}\right)+H_{p}^{q}\left(\mathbf{u}_{o}\right)\right)
$$

Thus $a_{0 p}^{0 q}$ are the expansion coefficients of the BRDF under fixed incident direction. The total squared energy of the BRDF is defined as the integral $\left|\rho_{b d}\left(\mathbf{u}_{i}, \mathbf{u}_{o}\right)\right|^{2}$ over its entire domain. Therefore the amount of signal energy captured by a single coefficient can be derived as follows.

$\int_{\Omega_{o}} \int_{\Omega_{i}}\left|\rho_{b d}\left(\mathbf{u}_{i}, \mathbf{u}_{o}\right)\right|^{2} d \omega_{i} d \omega_{o}=\sum_{n p m q} \sum_{n^{\prime} p^{\prime} m^{\prime} q^{\prime}} a_{n p}^{m q} a_{n^{\prime} p^{\prime}}^{m^{\prime} q^{\prime}} \int_{\Omega_{o}} \int_{\Omega_{i}} \mathscr{H}_{n p}^{m q}\left(\mathbf{u}_{i}, \mathbf{u}_{o}\right) \mathscr{H}_{n^{\prime} p^{\prime}}^{m^{\prime} q^{\prime}}\left(\mathbf{u}_{i}, \mathbf{u}_{o}\right) d \omega_{i} d \omega_{o}$

For a fixed incident direction, we have,

\footnotetext{
${ }^{1}$ Complex conjugate is dropped since we are dealing with the real form of the basis.
}

${ }^{2} H_{0}^{0}(\mathbf{u})=\frac{1}{\sqrt{2 \pi}}$ 


$$
\int_{\Omega_{o}} \int_{\Omega_{i}} \mathscr{H}_{n p}^{m q}\left(\mathbf{u}_{i}^{o}, \mathbf{u}_{o}\right) \mathscr{H}_{n^{\prime} p^{\prime}}^{m^{\prime} q^{\prime}}\left(\mathbf{u}_{i}^{o}, \mathbf{u}_{o}\right) d \omega_{i} d \omega_{o}=2 \pi \int_{\Omega_{o}} \mathscr{H}_{n p}^{m q}\left(\mathbf{u}_{i}^{o}, \mathbf{u}_{o}\right) \mathscr{H}_{n^{\prime} p^{\prime}}^{m^{\prime} q^{\prime}}\left(\mathbf{u}_{i}^{o}, \mathbf{u}_{o}\right) d \omega_{o}
$$

Using (7) and the orthonormality property of hemispherical harmonics (6), all terms in the integral vanishes except for $n=n^{\prime}, m=m^{\prime}, p=p^{\prime}$ and $q=q^{\prime}$, leading to,

$$
\left(N_{n p}^{m q}\right)^{2}\left[\left(H_{n}^{m}\left(\mathbf{u}_{i}^{o}\right)\right)^{2}+\left(H_{p}^{q}\left(\mathbf{u}_{o}\right)\right)^{2}+2 H_{n}^{m}\left(\mathbf{u}_{i}^{o}\right) H_{p}^{q}\left(\mathbf{u}_{o}\right) \delta_{n p} \delta_{m q}\right]
$$

Returning to (14), and using (11) and (12), we will have,

$$
\int_{\Omega_{o}}\left|\rho_{b d}\left(\mathbf{u}_{i}^{o}, \mathbf{u}_{o}\right)\right|^{2} d \omega_{o}=\frac{2}{\pi}\left(a_{00}^{00}\right)^{2}\left(N_{00}^{00}\right)^{2}+2 \sum_{p=1}^{\infty} \sum_{q=-p}^{p}\left(a_{0 p}^{0 q}\right)^{2}\left(N_{0 p}^{0 q}\right)^{2}\left[\frac{1}{2 \pi}+\left(H_{p}^{q}\left(\mathbf{u}_{o}\right)\right)^{2}\right]
$$

Considering the $N$ th order function approximation, such an approximation captures a certain amount of the function's total energy defined as the cumulative sum of energies maintained by individual harmonic terms, (this cumulative energy can also be referred to as function approximation accuracy). Thus the approximation accuracy (or the energy captured by the $N$ th order approximation) can be defined as,

$$
e_{N}= \begin{cases}\frac{\frac{2}{\pi}\left(a_{00}^{00}\right)^{2}\left(N_{00}^{00}\right)^{2}}{\int_{\Omega_{o}}\left|\rho_{b d}\left(\mathbf{u}_{i}^{o}, \mathbf{u}_{o}\right)\right|^{2} d \omega_{o}} & N=0 \\ \frac{\frac{2}{\pi}\left(a_{00}^{00}\right)^{2}\left(N_{00}^{00}\right)^{2}+2 \sum_{p=1}^{N} \sum_{q=-p}^{p}\left(a_{0 p}^{0 q}\right)^{2}\left(N_{0 p}^{0 q}\right)^{2}\left[\frac{1}{2 \pi}+\left(H_{p}^{q}\left(\mathbf{u}_{o}\right)\right)^{2}\right]}{\int_{\Omega_{o}}\left|\rho_{b d}\left(\mathbf{u}_{i}^{o}, \mathbf{u}_{o}\right)\right|^{2} d \omega_{o}} & N>0\end{cases}
$$

\section{Experimentation on Compact Description of BRDF Physical models}

The approximating orders of the Helmholtz HSH-based basis expansion of an arbitrary BRDF mainly depend on the form of the BRDF at hand, where smooth BRDFs require fewer coefficients for accurate representation compared to non-smooth/complex ones. In this section, we investigate the representation power of our proposed BRDF basis to approximate physical analytical models of BRDF such as Oren-Nayer BRDF [ $\square]$ and Cook-Torrance BRDF [ 日]. They are physically-based micro-facet models where the former models facets as perfectly diffuse while the latter is focused on glossy reflectance.

Consider a BRDF model with a parameter set $\mathscr{P}=\left\{p_{1}, p_{2}, \ldots, p_{d}\right\}$ whose incoming hemisphere being sampled, where $\mathscr{P}_{C T}=\left\{\rho_{d}, \rho_{s}, \mathrm{~m}, \mathrm{n}\right\}^{3}$ and $\mathscr{P}_{O N}=\left\{\rho_{d}, \sigma\right\}^{4}$ for CookTorrance and Oren-Nayer BRDFs respectively. The amount of energy captured by the $N$ th order approximation can be expressed as $e_{N}(\mathscr{P})=\int_{\Omega_{i}} e_{N}\left(\mathbf{u}_{i}, \mathscr{P}\right) d \omega_{i}$. Thus in order to study the effect of a specific parameter $p_{k}$, we can marginalize the approximation accuracy to become a function of the parameter $p_{k}$. In order to assess the representation power of our proposed BRDF basis, we sampled the parameter-set domain of Cook-Torrance based on the statistics for Torrance-Sparrow BRDF model, provided by the MERL/ETH skin reflectance database developed by Weyrich et al. [R], which was derived from measuring the skin reflectance of 156 subjects with varying age, gender and race. In our experimentation, we sampled the distribution of surface roughness $m$ and specular reflectivity $\rho_{s}$, where $\mu(\mathrm{m})=0.3032, \operatorname{std}(\mathrm{m})=0.0891, \mu\left(\rho_{s}\right)=0.3753$ and $\operatorname{std}\left(\rho_{s}\right)=0.1655$, while $\rho_{d}$ was taken as $1-\rho_{s}$ and skin refractive index $\mathrm{n}=1.4433$ according to [ $\left.\mathrm{\theta}\right]$. On the other hand, the domain of surface roughness parameter for the Oren-Nayar BRDF was uniformly sampled where $\sigma \in[0,1]$ where unit diffuse albedo is assumed. ${ }^{5}$

\footnotetext{
${ }^{3} \mathrm{n}$ is the classical refractive index from geometric optics, $\mathrm{m}$ is the root mean square slope of the micro-facets describing the surface's roughness, $\rho_{s}$ is the surface specular reflectivity and $\rho_{d}$ is the surface diffuse reflectivity.

${ }^{4} \sigma$ is the standard deviation of the Gaussian distribution used to represent surface roughness and $\rho_{d}$ is the surface diffuse reflectivity.

${ }^{5}$ We used Monte Carlo integration to evaluate the expansion coefficients of the BRDFs.
} 




(a)

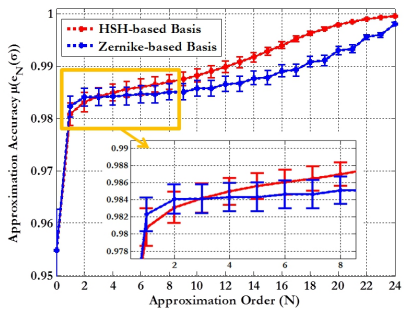

(b)

Figure 2: The Oren-Nayar BRDF approximation accuracy of the Helmholtz HSH-based basis as a function of the approximating order, where in (a) each curve corresponds to the average approximation accuracy over all sampled incident directions for several values of surface roughness, and in (b) it is compared to Zernike-based basis proposed by Koenderink et al. [ $\square$ ], where the mean and standard deviation were taken over $e_{N}(\sigma)$.

Fig. 2(a) shows the Oren-Nayar BRDF approximation accuracy of our Helmholtz HSHbased basis as a function of the approximating order $N$ for several values of surface roughness $\sigma$. Each curve corresponds to the average approximation accuracy over sampled incident directions, or more formally $e_{N}(\sigma)=\int_{\Omega_{i}} e_{N}\left(\mathbf{u}_{i}, \sigma\right) d \omega_{i}$. It can be observed that; (1) for zero surface roughness, the Oren-Nayar BRDF boils to a Lambertian model whose total energy is captured by the zero-order approximation, (2) as the surface roughness increase, zero-order approximation captures less energy, thus with rougher surfaces, higher orders are needed to maintain a specific level of accuracy, (3) beyond the sixth order, excluding smooth surfaces, the surface roughness parameter has insignificant impact on the Oren-Nayar BRDF approximation accuracy. Fig. 2(b) shows mean and standard deviation of $e_{N}(\sigma)$ of the OrenNayar BRDF for the Helmholtz HSH-based basis in contrast to the basis based on Zernike polynomials, as functions of the approximation order $N$. It can be observed that (1) both basis have the same zero-order accuracy, since the zero-order basis corresponds to the BRDF DC component, (2) Zernike-based basis outperforms HSH-based basis up to the second order, after which HSH-based basis performance grows faster than that of the Zernike-based basis. This emphasizes the effect of reflectance basis at all combination of orders and degrees as in the case of HSH when compared to Zernike polynomials.

In case of Cook-Torrance BRDF, the grazing incident angles represents a problem in the BRDF construction, where the shape of the BRDF varies from Lambertian-like shape in case of very low specular reflectivity to specular-lobe in case of high specular reflectivity. This introduces a source of discontinuity/non-smoothness in the BRDF. Since harmonicbased basis are compact in the frequency domain, they are capable of representing smooth BRDFs with fewer non-zero coefficients when compared to complex BRDFs. It has been known that both spherical harmonics and Zernike polynomials require large number of basis to represent specular BRDFs []]. Since HSH is an adapted version of SH, it is anticipated to behave similarly. Rusinkiewicz [ $[2]$ proposed a change of variables to re-parameterize the BRDF in order to yield efficient decompositions/representations of specular BRDFs, this will be handled in our future efforts. In this paper, we investigate our reflectance basis in the original/intuitive parameterizations of incident and outgoing angles. For purposes of comparison to Zernike-based basis, we have two situations; (1) non-grazing and (2) grazing incident angles, where throughout our experimentations, an incident angle of $\theta_{i}>\frac{7 \pi}{18}$ was considered to be a grazing incident angle. Fig. 3 shows an example of fitting HSH-based basis versus the Zernike-based ones on a Cook-Torrance BRDF with high surface specular 


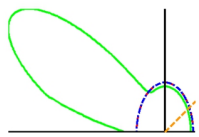

$\mathrm{N}=0$



$N=4$



$\mathrm{N}=1$

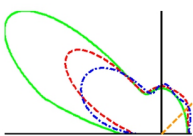

$N=8$

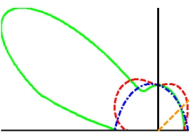

$\mathrm{N}=2$

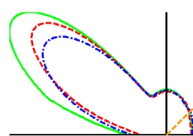

$N=16$



--- Reconstructed BRDF - Zerrike-based Basis

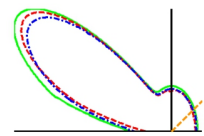

$\mathrm{N}=24$

Figure 3: Example of fitting HSH-based basis (dashed red) versus the Zernike-based ones (dashed blue) on a Cook-Torrance BRDF (solid green) with high surface specular reflectivity, where $\rho_{s}=$ $0.458, \mathrm{~m}=0.125, \theta_{i}=\pi / 3, \phi_{i}=0$. Notice how HSH-based basis provide better fitting at lower orders when compared to the Zernike-based basis. For visualization purposes, the incident plane is plotted and dashed orange line represents the light incident direction.

reflectivity.

Fig. 4 shows the approximation accuracy of the Cook-Torrance BRDF in case of nongrazing and grazing incident angles, where each curve shows the mean and standard deviation of $e_{N}\left(\rho_{s}, \mathrm{~m}\right)$. We can observed that the HSH-based basis functions provide better representation when compared to the Zernike-based ones in case of non-grazing incident angles, however, this is not the case for grazing angles where specularity perturbs the smoothness of the BRDF, one way to tackle such a problem is to re-parameterize the BRDF as proposed by [Q]].

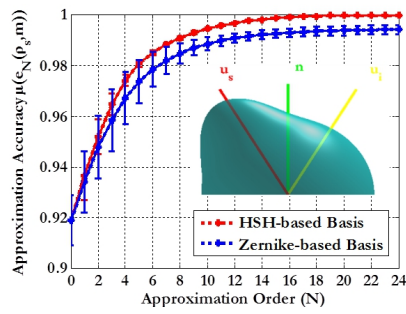

(a)

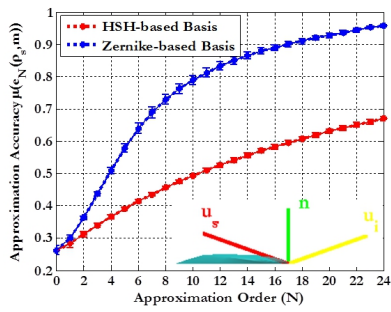

(b)

Figure 4: The Cook-Torrance BRDF approximation accuracy of the Helmholtz HSH-based basis compared to Zernike-based basis proposed by Koenderink et al. [ $\square$ ] as a function of the approximating order, where the mean and standard deviation were taken over $e_{N}\left(\rho_{s}, \mathrm{~m}\right)$ : (a) non-grazing incident angles, where $\theta_{i} \in\left[0, \frac{7 \pi}{18}\right]$, (b) grazing incident angles, where $\theta_{i}>\frac{7 \pi}{18}$. An example of the BRDF is visualized for the two cases where $\mathrm{m}=0.2, \mathrm{n}=1.44, \rho_{s}=0.6$ and $\rho_{d}=0.4$.

In the following, discussion is restricted to the case of non-grazing incident angles. Fig. 5 illustrates the effect of surface roughness of Cook-Torrance model on the approximation accuracy of HSH-based basis, Fig. 5(a), versus Zernike-based ones, Fig. 5(b), where each curve represents the average approximation accuracy taken over several values of surface specular reflectivity. In general, the rougher the surface, the less specular it looks, this results in having more energy captured at lower approximation orders. In the close-up views, it can be observed that the effect of surface roughness on the approximation accuracy decreases at lower orders in case of HSH-based basis when compared to the Zernike-based ones. In other words, Zernike-based basis are more sensitive to surface roughness parameter of the Cook-Torrance model.

Fig. 6 shows the effect of surface specular reflectivity on the approximation accuracy of 


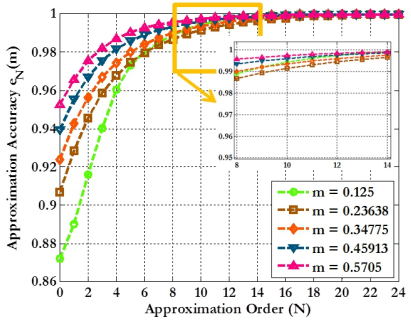

(a)

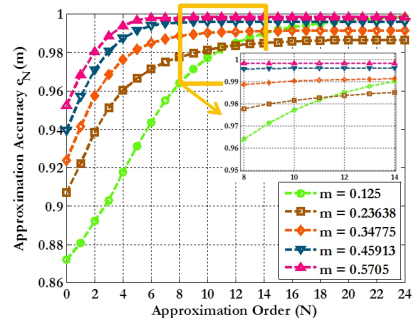

(b)

Figure 5: The Cook-Torrance BRDF approximation accuracy of (a) our Helmholtz surface reflectance basis, (b) Zernike-based basis, as a function of the approximating order, where each curve corresponds to the average approximation accuracy over all sampled incident directions for several values of surface roughness.

HSH-based basis Fig. 6(a) versus that of Zernike-based basis Fig. 6(b), where each curve represents the average approximation accuracy taken over several values of surface roughness. With lower specular reflectivity, the performance of the approximation accuracy tends toward the Lambertian case, where all the function's energy is captured with the zero-order coefficient. This performance degrades with more specular reflectivity. It can be observed that our Helmholtz basis captures more function energy at lower orders when compared to Zernike-based ones. In the close-up views, we can notice the sensitivity of the Zernike-based basis to the specular reflectivity, while on the other hand the effect of the specular reflectivity on the approximation accuracy decreases at lower orders in case of HSH-based basis.

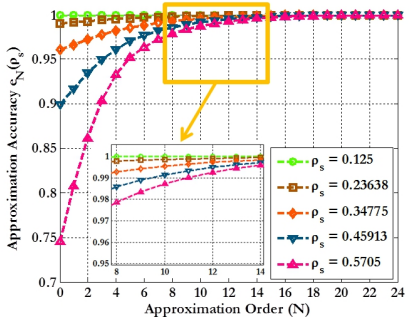

(a)

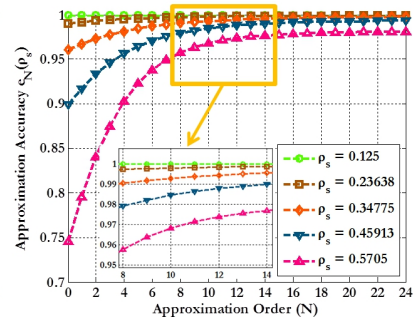

(b)

Figure 6: The Cook-Torrance BRDF approximation accuracy of (a) our Helmholtz surface reflectance basis, (b) Zernike-based basis, as a function of the approximating order, where each curve corresponds to the average approximation accuracy over all sampled incident directions for several values of specularity reflectivity.

\section{Conclusion}

In this paper, we proposed a complete, orthonormal basis to provide a compact and efficient representation for surface bidirectional reflectance distribution function (BRDF), which is defined on the cartesian product of two hemispheres. Our basis, which are defined in terms of hemispherical harmonics (HSH), preserve the Helmholtz reciprocity property of BRDFs while avoid the computational complexity inherited from Zernike polynomials that are usually used to construct hemispherical basis. We validated our basis using micro-facet physical BRDF models: Oren-Nayar for diffuse rough surfaces and Cook-Torrance for specular rough surfaces. Based on the fact that associated Legendre polynomials are defined for all combinations of polynomial order and degree in contrast to Zernike polynomials, HSHbased basis showed high BRDF approximation accuracy at lower orders. It was observed 
that the significance of BRDF parameters decayed with the approximation order versus the case of Zernike-based basis. The grazing incident angles represented a problem in case of Cook-Torrance BRDF which introduces a source of non-smoothness in the BRDF, where the HSH-based basis functions provide better representation when compared to the Zernikebased ones in case of non-grazing incident angles, however, this is not the case for grazing angles. Ongoing efforts are directed towards (1) handling grazing incident angles using BRDF re-parameterization and (2) experimenting our basis to model scattered BRDF measurements, e.g. the CUReT database [0].

\section{References}

[1] N. Alldrin, T. Zickler, and D. Kriegman. Photometric stereo with non-parametric and spatially-varying reflectance. In Computer Vision and Pattern Recognition, 2008. CVPR 2008. IEEE Conference on, pages 1 -8, june 2008.

[2] R. Basri and D. W. Jacobs. Lambertian reflectance and linear subspaces. IEEE Transactions on Pattern Analysis and Machine Intelligence, 25(2):218-233, Feb 2003.

[3] R. Basri, D. Jacobs, and I. Kemelmacher. Photometric stereo with general, unknown lighting. International Journal of Computer Vision, 72(3):239-257, 2007.

[4] A. B. Bhatia and E. Wolf. On the circle polynomials of zernike and related orthogonal sets. In Mathematical Proceedings of the Cambridge Philosophical Society, number 50.

[5] C. D. H. Chisholm. Group theoretical techniques in quantum chemistry. Academic Press, London, New York, 1976. ISBN 0121729508.

[6] R. L. Cook and K. E. Torrance. A reflectance model for computer graphics. ACM Transaction of Graphics, 1(1):7-24, 1982.

[7] K. Dana, B. Ginneken, S. Nayar, and J. Koenderink. Reflectance and texture of realworld surfaces. ACM Transactions on Graphics, 18(1):1-34, January .

[8] E. Eibenberger and E. Angelopoulou. Beyond the neutral interface reflection assumption in illuminant color estimation. In 17th IEEE International Conference on Image Processing (ICIP), number 50.

[9] P. Gautron, J. Krivat'nek, S.N. Pattanaik, and K. Bouatouch. A novel hemispherical basis for accurate and efficient rendering. In Proc. Eurographics Symposium on Rendering, 2004.

[10] B.K.P. Horn. Shape from Shading, A Method for Obtaining the Shape of a Smooth Opaque Object from One View. PhD thesis, Massachusetts Inst. of Technology, Cambridge, Massachusetts, 1970.

[11] I. Kemelmacher-Shlizerman and R. Basri. 3d face reconstruction from a single image using a single reference face shape. IEEE Transactions on Pattern Analysis and Machine Intelligence (PAMI), 33(2):394-405, 2010.

[12] J. Koenderink and A. van Doorn. Phenomenlogical description of bidirectional surface reflection. Journal of the Optical Society of America, 15(11):2903-2912, 1998. 
[13] J. Koenderink, A. Van Doorn, and M. Stavridi. Bidirectional reflection distribution function expressed in terms of surface scattering modes. In ECCV, pages 28-39, 1996.

[14] O. A. Makhotkin. Analysis of radiative transfer between surfaces by hemispherical harmonics. Journal of Quantitative Spectroscopy and Radiative Transfer, 56(6):869879, 1996.

[15] P. Nillius. Image Analysis using the Physics of Light Scattering. Doctoral Thesis, KTH Numerical Analysis and Computer Science, Stockhold, Sweden, 2004.

[16] P. Nillius, J. Sullivan, and A. A. Argyros. Shading models for illumination and reflectance invariant shape detectors. In In Proc. IEEE Computer Vision and Pattern Recognition (CVPR08), Anchorage, June 2008.

[17] M. Oren and S. K. Nayar. Generalization of lambert's reflectance model. In Computer Graphics, 28(Annual Conference Series), pages 239-246, 1994.

[18] R. Ramamoorthi. A Signal-Processing Framework for Forward and Inverse Rendering. Ph.D. Dissertation. Stanford University, Stanford, CA, USA, Advisor(s) P. Hanrahan, 2002.

[19] H. Rara and A. Farag S. Elhabian, T. Starr. Model-based shape recovery from single images of general and unknown lighting. In 16th IEEE International Conference on Image Processing (ICIP2009), pages 517-520, 7-10 Nov. 2009.

[20] H. Rara, S. Elhabian, and A. Farag T. Starr. 3d face recovery from intensities of general and unknown lighting using partial least squares. In 17th IEEE International Conference on Image Processing (ICIP2010), Sept. 2010.

[21] K. F. Riley, M. P. Hobson, and S. J. Bence. Mathematical Methods for Physics and Engineering. Cambridge University Press, 2nd edition, 2002. Ch19, pp. 670.

[22] Szymon Rusinkiewicz. A new change of variables for efficient BRDF representation. In Rendering Techniques (Proc. Eurographics Workshop on Rendering), June 1998.

[23] S. Sarkar. Usf humanid 3d face database. University of South Florida, Tampa, FL, USA.

[24] P. P. Sloan, J. Hart J. Hall, and J. Snyder. Clustered principal components for precomputed radiance transfer. ACM Transactions of Graphics, 22(3):382-391, 2003.

[25] S. H. Westin, J. R. Arvo, and K. E. Torrance. Predicting reflectance functions from complex surfaces. In Proc. of SIGGRAPH, ACM Press, pages 255-264, 1992.

[26] T. Weyrich, W. Matusik, H. Pfister, B. Bickel, C. Donner, C. Tu, J. McAndless, J. Lee, A. Ngan, H. W. Jensen, and M. Gross. Analysis of human faces using a measurementbased skin reflectance model. ACM Trans. Graph., 25:1013-1024, July 2006.

[27] J. C. Wyant and K. Creath. Basic wavefront aberration theory for optical metrology. In Applied optics and Optical Engineering, Academic Press, Inc. 